\title{
Landing of VTOL UAVs Using a Stationary Robot Manipulator: A New Approach for Coordinated Control
}

\author{
Moritz Maier and Konstantin Kondak
}

\begin{abstract}
This paper deals with the landing of VTOL UAVs under severe conditions. We present a novel system based on a robot manipulator, which is used to support a UAV in the last, most challenging, landing phase. In this phase, the aerial vehicle is connected to the robot by means of a universal hinge. This allows to decouple the UAV's orientation from the robot's end-effector orientation. The main contribution of the paper is a new control approach for the whole system composed of a VTOL UAV and a manipulator. A combination of a backstepping controller accounting for the UAV's dynamics with an impedance controller for the manipulator is used for coordinated control of the whole system. The proposed approach allows to independently control position and orientation of a VTOL UAV, whereby an arbitrary stable attitude controller can be used for the flying vehicle. The advantage of the presented approach is that the interaction forces between robot and UAV are taken into account explicitly and that a Lyapunov stability proof for the UAV subsystem can be derived directly. Robustness and performance of the control approach are investigated in simulation and experiments. The experimental results for an AR.Drone quadrotor and a DLR/KUKA light-weight robot with seven degrees-of-freedom are presented in the paper.
\end{abstract}

\section{INTRODUCTION}

Due to their vertical take-off and landing (VTOL) capabilities, rotary-wing unmanned aerial vehicles (UAVs) are nowadays widely used, e.g. for search-and-rescue, inspection, and aerial photography applications [1]. For some missions, landing the vehicle can become a crucial task due to severe weather conditions or especially if a touch-down on a moving platform is required. Challenging are for instance heavy and unsteady wind gusts near ground or platform movements, which are hard to predict, e.g. ship motions [2].

In order to recover the UAV rotorcraft safely under severe conditions, the control strategy as well as the hardware of the flying system need to be adjusted. Available approaches range from incorporating wind observance and platform motion in the controller design [3]-[5] through utilizing special visual sensor equipment [6,7] to using supporting ground systems, such as a hexapod robot [8] or a tether $[9,10]$. A hexapod, as presented in [8], is able to compensate platform motion to a certain extend, but it is limited in its operating range and does not provide any clamping mechanism to fix the UAV and prevent it from sliding. In contrast, using a tether as in [9] and [10] offers a much larger operating range, fixes the UAV to the platform, and does not require GPS position information [10]. Nevertheless, the rope angles have to be measured and a device for providing desired rope force

moritz.maieredlr.de, German Aerospace Center (DLR), Robotics and Mechatronics Center (RMC), Münchener Straße 20, 82234 Weßling, Germany

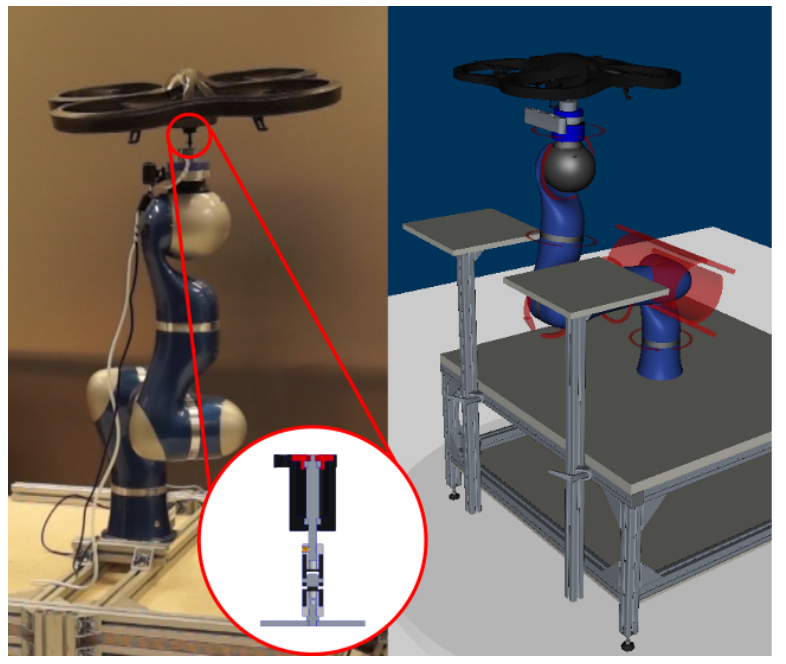

Fig. 1: Novel VTOL UAV landing system demonstrator in the DLR Flying Robots lab with custom-built universal hinge (left) and screenshot from the simulation and visualization environment (right).

is needed. Furthermore, the tether can only transfer forces in one direction and is therefore not able to increase the lifting force of the VTOL UAV.

Hence, we propose a novel robotic recovery system, which consists of a serial robotic manipulator that is used to capture the UAV in-flight (see Fig. 1). To the knowledge of the authors, such a system has not been studied up to now. It is advantageous to existing approaches since it

- does not require additional power of the UAV's engine,

- can also be used for take-off while the possible take-off weight is increased when a forward velocity is induced,

- and is able to compensate platform motion within the boundaries of the robot's dexterous workspace.

We divide the complete landing maneuver into three phases: approach, in-flight capturing, and physical interaction. Within this paper, we focus on the last phase of the landing maneuver during which the manipulator is in contact with the VTOL UAV and assists it in order to land it safely on the ground. The UAV is connected to the robot via a universal hinge, as shown in Fig. 1, such that the UAV can maintain a desired attitude independent from the robot's endeffector orientation.

The goal is to control the position of the UAV using the robot manipulator in a compliant manner. A well-known approach for physical interaction of a robot with the environment is impedance control [11]. For the task specified above, the performance of impedance control highly depends on the model of the environment. We therefore extend the classical 
impedance controller to include the dynamics of the attitudecontrolled VTOL UAV. Instead of a linear mass-springdamper system, we derive a backstepping control law [12] for the force that is exerted by the robot on the UAV. The VTOL UAV and the manipulator are considered as two subsystems and it is assumed that the small-scale aerial vehicle has minor influence on the robot. In order to compensate the influence of the acceleration of the robot's end-effector on the orientation of the UAV, the backstepping controller generates an additional torque that is sent to the UAV and added to the torque of the onboard attitude controller. We demonstrate that this strategy allows a coordinated control of the robot and the UAV such that the UAV follows a desired attitude and position trajectory.

For experiments we use the demonstrator shown in Fig. 1. Its main component is a DLR/KUKA light-weight robot (LWR, [11], p. 3) on a fixed base. At the end-effector, a camera system for visually tracking the UAV, a force-torque sensor, and an electromagnet are mounted. The magnet is utilized for attaching and detaching the flying system to a metal plate at the end of the universal hinge.

This work is structured as follows: in Section II, the concept of impedance control as well as the nonlinear dynamic models of a serial robot manipulator and of a quadrotor-type unmanned aerial vehicle are introduced and employed for controller design in Section III. In Section IV, the feasibility and performance of the new coordinated control approach for the physical interaction task is evaluated in experiments. Finally, the paper is concluded and an outlook is given in Section V.

\section{RoBOT AND UAV MODELING}

In this section, first a dynamic model of a robot manipulator and the concept of impedance control is presented. Then, the rigid-body model of a quadrotor is derived, which is used in Section III for the backstepping controller design.

\section{A. Impedance Controlled Serial Manipulator}

The dynamics in joint coordinates $\phi \in \boldsymbol{\Phi}^{n}$ of a serial robotic manipulator with $n$ joints are given by ([11], p. 29)

$$
\boldsymbol{M}(\phi) \ddot{\phi}+C(\phi, \dot{\phi}) \dot{\phi}+\boldsymbol{g}(\phi)=\tau_{j}+\tau_{e x t},
$$

where $\phi, \boldsymbol{M}_{n \times n}, \boldsymbol{C}_{n \times n}$, and $\boldsymbol{g}_{n \times 1}$ are the joint position, the mass matrix, the Coriolis/centrifugal matrix, and the vector of gravity terms, respectively. We assume that the joint torques $\boldsymbol{\tau}_{j}$ are controlled directly. The relationship between the joint angles $\phi$ and the Cartesian end-effector coordinates $\boldsymbol{x} \in \mathbb{R}^{6}$ is given by the forward kinematics of the robot $\boldsymbol{f}: \boldsymbol{\Phi} \rightarrow \mathbb{R}^{6}$. For a redundant manipulator with $n>6$, e.g. the LWR, the external forces at the end-effector $\boldsymbol{F}_{\text {ext }}$ can be related to joint torques by [13]

$$
\boldsymbol{\tau}_{\text {ext }}=\mathcal{J}(\phi)^{T} \boldsymbol{F}_{\text {ext }}+\boldsymbol{\tau}_{n s p},
$$

using the Jacobian $\mathcal{J}(\phi)=\frac{\partial \boldsymbol{f}(\phi)}{\partial \phi}$. Therein, $\boldsymbol{\tau}_{n s p}$ comprises torques in the nullspace of the robot, i.e. joint torques that do not influence the end-effector motion. The dynamics (1) of

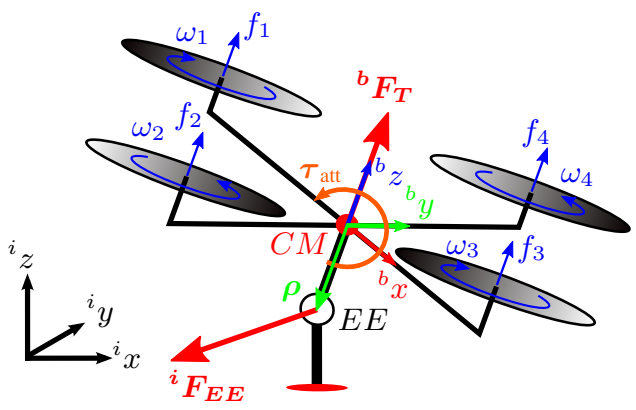

Fig. 2: Quadrotor model.

a redundant manipulator can be rewritten in Cartesian endeffector coordinates as

$$
\boldsymbol{\Lambda}(\phi) \ddot{\boldsymbol{x}}+\boldsymbol{\mu}(\phi, \dot{\boldsymbol{\phi}}) \dot{\boldsymbol{x}}+\boldsymbol{F}_{g}(\phi)=\boldsymbol{F}_{\tau}+\boldsymbol{F}_{\text {ext }},
$$

with $\boldsymbol{\tau}_{j}=\mathcal{J}(\phi)^{T} \boldsymbol{F}_{\tau}+\boldsymbol{\tau}_{n s p}$. In the case $n>6$, the inertial properties $\boldsymbol{\Lambda}(\phi), \boldsymbol{\mu}(\boldsymbol{\phi}, \dot{\boldsymbol{\phi}}) \dot{\boldsymbol{x}}$, and $\boldsymbol{F}_{g}(\phi)$ vary not only with the configuration $\boldsymbol{x}$, but also with the manipulator posture $\phi$. The pseudo kinetic energy matrix is obtained from

$$
\boldsymbol{\Lambda}(\phi)=\mathcal{J}(\phi)^{-T} \boldsymbol{M}(\phi) \mathcal{J}(\phi)^{-1},
$$

for which a generalized inverse of the Jacobian $\mathcal{J}(\phi)$ can be used [13]. In classical Cartesian impedance control without energy shaping, the desired dynamical behaviour is defined with $\boldsymbol{x}_{e}=\boldsymbol{x}-\boldsymbol{x}_{d}$ as ([11], p. 35)

$$
\boldsymbol{\Lambda}(\phi) \ddot{\boldsymbol{x}}_{e}+\left(\boldsymbol{\mu}(\boldsymbol{\phi}, \dot{\boldsymbol{\phi}})+\boldsymbol{D}_{d}\right) \dot{\boldsymbol{x}}_{e}+\boldsymbol{K}_{d} \boldsymbol{x}_{e}=\boldsymbol{F}_{e x t},
$$

for which desired damping $\boldsymbol{D}_{d}>\mathbf{0}$, and stiffness $\boldsymbol{K}_{d}>\mathbf{0}$ can be selected. $\boldsymbol{x}_{d}$ is the desired end-effector pose. The impedance controller can be derived from (5) and (3), as

$$
\begin{aligned}
\boldsymbol{\tau}_{j}= & \boldsymbol{g}(\boldsymbol{\phi})+\boldsymbol{\tau}_{n s p}+\mathcal{J}(\boldsymbol{\phi})^{T}\left(\boldsymbol{\Lambda}(\boldsymbol{\phi}) \ddot{\boldsymbol{x}}_{d}+\boldsymbol{\mu}(\boldsymbol{\phi}, \dot{\boldsymbol{\phi}}) \dot{\boldsymbol{x}}_{d}\right) \\
& -\mathcal{J}(\boldsymbol{\phi})^{T}\left(\boldsymbol{K}_{d} \boldsymbol{x}_{e}+\boldsymbol{D}_{d} \dot{\boldsymbol{x}}_{e}\right)
\end{aligned}
$$

with $\boldsymbol{I}$ being the identity matrix. In contrast to (6), we design a more specific control law, which incorporates the dynamical model of the VTOL UAV (see Section III).

\section{B. Attitude Controlled VTOL UAV}

For modeling the unmanned aerial vehicle, we use a rigidbody model, as presented in [14]. The rotational dynamics written in the body-fixed frame $b$ and the translational dynamics written in the inertial frame $i$ (see Fig. 2) can be combined as

$$
\left[\begin{array}{cc}
\boldsymbol{J} & \mathbf{0} \\
\mathbf{0} & m \boldsymbol{I}
\end{array}\right]\left(\begin{array}{c}
{ }^{b} \dot{\boldsymbol{\omega}} \\
{ }^{i} \dot{\boldsymbol{v}}
\end{array}\right)=\left(\begin{array}{c}
-\boldsymbol{S}\left({ }^{b} \boldsymbol{\omega}\right) \boldsymbol{J}^{b} \boldsymbol{\omega}+\boldsymbol{\tau}_{u a v} \\
-m g \boldsymbol{e}_{3}+\boldsymbol{F}_{u a v}
\end{array}\right),
$$

where ${ }^{b} \boldsymbol{\omega}$ and ${ }^{i} \boldsymbol{v}$ are the rotational and translational velocity of the UAV, respectively. $\boldsymbol{S}(\cdot)$ is used to denote a skewsymmetric matrix, i.e.

$$
\boldsymbol{S}(\boldsymbol{\omega})=\left[\begin{array}{ccc}
0 & -\omega_{3} & \omega_{2} \\
\omega_{3} & 0 & -\omega_{1} \\
-\omega_{2} & \omega_{1} & 0
\end{array}\right]
$$

In (7), $m$ is the collective mass of the UAV, $\boldsymbol{J}$ its inertia, $g$ is the gravitational acceleration, and $\boldsymbol{e}_{3}=\left(\begin{array}{lll}0 & 0 & 1\end{array}\right)^{T}$ is a unit vector. All forces acting on the UAV and all torques about its body-fixed axes are summed up in $\boldsymbol{F}_{\text {uav }}$ and $\tau_{\text {uav }}$, respectively. Note, that the ${ }^{b} z$-axis points upwards 


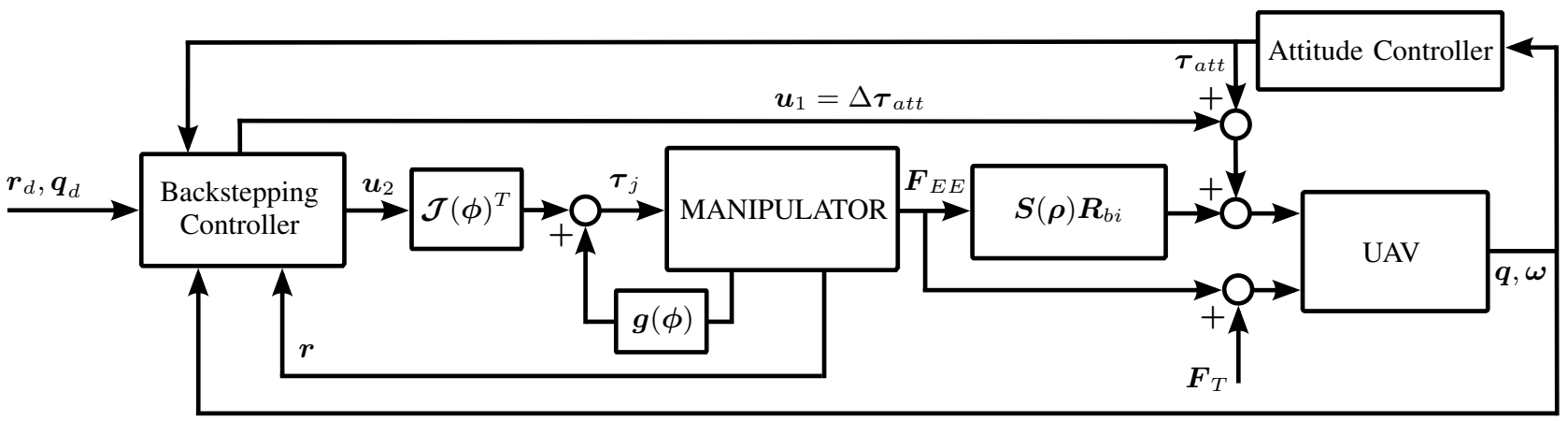

Fig. 3: Proposed control structure for coordinated control of a robot manipulator and a VTOL UAV.

since this can be beneficial for control design, as shown in [14], and coincides with the convention used for robotic end-effectors. As soon as robot and UAV are connected via the rotational hinge, the UAVs center of mass $C M$ will move only relatively to the pivot point $E E$, as depicted in Fig. 2, with a lever arm from $C M$ to $E E$ defined as ${ }^{b} \boldsymbol{\rho}=\left[\begin{array}{lll}0 & 0 & l\end{array}\right]^{T}$. A torque balance about $C M$ yields

$$
\boldsymbol{\tau}_{u a v}=\boldsymbol{\tau}_{a t t}+\boldsymbol{S}\left({ }^{b} \boldsymbol{\rho}\right) \boldsymbol{R}_{b i}{ }^{i} \boldsymbol{F}_{E E} .
$$

Therein, $\boldsymbol{\tau}_{a t t}$ is the torque produced by the rotors of the UAV in order to stabilize a desired attitude and $\boldsymbol{R}_{i b}$ is a rotation matrix, that transfers a vector given in the body-fixed frame $b$ to the inertial frame $i$. Similarly, the external forces are obtained from a force balance as

$$
\boldsymbol{F}_{\text {uav }}=f_{T} \boldsymbol{R}_{b i}^{T} \boldsymbol{e}_{3}+{ }^{i} \boldsymbol{F}_{E E},
$$

wherein $f_{T}=\sum_{i=1}^{4} f_{i}$ is the thrust force of the UAV which is assumed to be always perpendicular to the $\left({ }^{b} x,{ }^{b} y\right)$-plane (see Fig. 2) and ${ }^{i} \boldsymbol{F}_{E E}$ is the force acting at the end-effector. Inserting (9) and (10) in (7) yields the equation of motion of the UAV connected to the robot manipulator via a universal hinge:

$$
\begin{aligned}
\underbrace{\left[\begin{array}{cc}
\boldsymbol{J} & \mathbf{0} \\
\mathbf{0} & m \boldsymbol{I}
\end{array}\right]}_{\boldsymbol{M}_{\text {uav }}}\left(\begin{array}{c}
{ }^{b} \dot{\boldsymbol{\omega}} \\
{ }^{i} \dot{\boldsymbol{v}}
\end{array}\right) & =\underbrace{\left(\begin{array}{c}
-\boldsymbol{S}^{b}\left({ }^{b} \boldsymbol{\omega}\right) \boldsymbol{J}^{b} \boldsymbol{\omega}+\boldsymbol{\tau}_{a t t} \\
-m g \boldsymbol{e}_{3}+f_{T} \boldsymbol{R}_{b i}^{T} \boldsymbol{e}_{3}
\end{array}\right)}_{\boldsymbol{a}_{\text {uav }}} \\
& +\underbrace{\left[\begin{array}{c}
\boldsymbol{S}\left({ }^{b} \boldsymbol{\rho}\right) \boldsymbol{R}_{b i} \\
\boldsymbol{I}
\end{array}\right]}_{\boldsymbol{B}_{\text {uav }}}{ }^{i} \boldsymbol{F}_{E E} .
\end{aligned}
$$

In the remainder of this work, we denote the mass matrix of the VTOL UAV as $\boldsymbol{M}_{\text {uav }}$, the input matrix as $\boldsymbol{B}_{\text {uav }}$, and the rest of the right-hand side of (11) as $\boldsymbol{a}_{\text {uav }}$ in order to simplify the notation. It is assumed, that the UAV is equipped with an arbitrary attitude controller, which generates the torque $\boldsymbol{\tau}_{a t t}$. Furthermore, $f_{T}$ will be set to a predefined value or can alternatively be used for height control.

\section{Controller Design}

We want to use the robot manipulator to guide the UAV to a desired position and attitude. Therefore, we design a controller, as depicted in Fig. 3, taking into account the rotational and translational dynamics of the attitude con- trolled UAV rotorcraft. We use the backstepping methodology ([12], p. 589) and show that this leads to a controller for the UAV subsystem, for which asymptotic closed-loop stability can be guaranteed.

\section{A. Control Input and Quaternion Error}

The input matrix $\boldsymbol{B}_{u a v}$ in (11) is not invertible due to the underactuation of the system, which prevents this model from being directly utilized for nonlinear backstepping controller design. Therefore, we append an additional torque input $\Delta \tau_{a t t}$, which is sent to the UAV and added to $\tau_{a t t}$, such that the control input becomes

$$
\boldsymbol{u}=\left(\begin{array}{ll}
\boldsymbol{u}_{1} & \boldsymbol{u}_{2}
\end{array}\right)^{T}=\left(\begin{array}{ll}
\Delta \boldsymbol{\tau}_{a t t} & { }^{i} \boldsymbol{F}_{E E}
\end{array}\right)^{T} .
$$

Hence, the system becomes fully actuated and the augmented input matrix $\overline{\boldsymbol{B}}_{\text {uav }}$ is invertible with

$$
\overline{\boldsymbol{B}}_{u a v}=\left[\begin{array}{cc}
\boldsymbol{I} & \boldsymbol{S}\left({ }^{b} \boldsymbol{\rho}\right) \boldsymbol{R}_{b i} \\
\mathbf{0} & \boldsymbol{I}
\end{array}\right] .
$$

The Cartesian position error $\boldsymbol{r}_{e}=\boldsymbol{r}_{d}-\boldsymbol{r}$ is defined as the difference between desired position $\boldsymbol{r}_{d}$ and current position $\boldsymbol{r}$ of the end-effector $E E$. In order to avoid representation singularities, we use quaternions $\boldsymbol{q}=\left(\begin{array}{cccc}\eta & \epsilon_{1} & \epsilon_{2} & \epsilon_{3}\end{array}\right)^{T}$ for attitude parametrization. Therefore, the attitude kinematics for the UAV can be derived as

$$
\dot{\boldsymbol{q}}=\frac{1}{2}\left[\begin{array}{c}
-\boldsymbol{\epsilon}^{T} \\
\eta \boldsymbol{I}_{3 \times 3}+\boldsymbol{S}(\boldsymbol{\epsilon})
\end{array}\right]{ }^{b} \boldsymbol{\omega}=\frac{1}{2} \boldsymbol{Q}_{q}(\boldsymbol{q})^{b} \boldsymbol{\omega} .
$$

The attitude error $\boldsymbol{q}_{e}$ is defined using quaternion multiplication [15], hence

$$
\boldsymbol{q}_{e}=\left(\begin{array}{ll}
\eta_{e} & \boldsymbol{\epsilon}_{e}
\end{array}\right)^{T}=\boldsymbol{q}_{d}^{-1} \otimes \boldsymbol{q}=\left[\begin{array}{ll}
\boldsymbol{q}_{d}^{-1} & \boldsymbol{Q}_{q}\left(\boldsymbol{q}_{d}^{-1}\right)
\end{array}\right] \boldsymbol{q},
$$

which is equal to the rotation from the desired attitude $\boldsymbol{q}_{d}$ to the current attitude $\boldsymbol{q}$.

\section{B. Backstepping Controller Design}

We derive the controller for the system depicted in Fig. 4 following the procedure presented in [16], but augment it to include both rotational and translational dynamics of the VTOL UAV. The controller combines UAV orientation and position control and the latter is used in Section III-C to extend the classical impedance controller (6).

The first backstepping variable $z_{1}$ is chosen to be

$$
\boldsymbol{z}_{1}=\left(\begin{array}{ccc}
1-\left|\eta_{e}\right| & \boldsymbol{\epsilon}_{e} & \boldsymbol{r}_{e}
\end{array}\right)^{T} \text {. }
$$




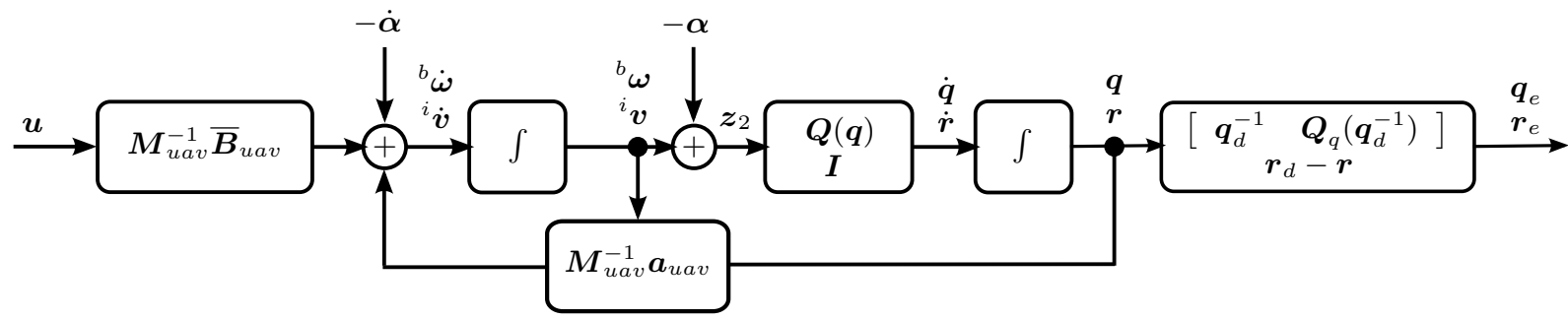

Fig. 4: Dynamics of the UAV subsystem as flow diagram for backstepping controller design.

The term $1-\left|\eta_{e}\right|$ is used in order to avoid unwinding [17]. The first derivative of (16) with respect to time is

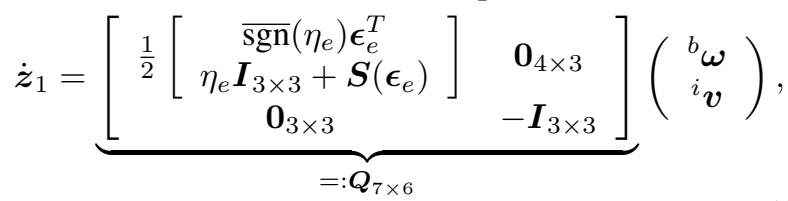

wherein $\overline{\operatorname{sgn}}(c)$ is a modified signum function defined as

$$
\overline{\operatorname{sgn}}(c)=\left\{\begin{array}{rl}
-1, & c<0 \\
1, & c \geq 0
\end{array} .\right.
$$

Next, we introduce the Lyapunov function candidate

$$
V_{1}=\frac{k_{3}}{2} \boldsymbol{z}_{1}^{T} \boldsymbol{z}_{1}>0
$$

which is positive definit for $k_{3}>0$. Its derivative with respect to time is

$$
\dot{V}_{1}=k_{3} z_{1}^{T} \dot{z}_{1}=k_{3} z_{1}^{T} \boldsymbol{Q}\left({ }^{b} \boldsymbol{\omega} \quad{ }^{i} \boldsymbol{v}\right)^{T} .
$$

Using a virtual control input $\boldsymbol{\alpha}$, we define the second backstepping variable $z_{2}$ to be

$$
\boldsymbol{z}_{2}=\left(\begin{array}{ll}
{ }^{b} \boldsymbol{\omega} & { }^{i} \boldsymbol{v}
\end{array}\right)^{T}-\boldsymbol{\alpha}
$$

An intermediate control law, that preserves asymptotic stability for the equilibrium ${ }^{b} \boldsymbol{\omega}={ }^{i} \boldsymbol{v}=\mathbf{0}$ of (21) is

$$
\boldsymbol{\alpha}=-\boldsymbol{K}_{1} \boldsymbol{Q}^{T} \boldsymbol{z}_{1}
$$

Therein, $\boldsymbol{K}_{1}>0$ is a positive definit diagonal gain matrix. Hence, (20) becomes

$$
\dot{V}_{1}=\underbrace{-k_{3} \boldsymbol{z}_{1}^{T} \boldsymbol{Q} \boldsymbol{K}_{1} \boldsymbol{Q}^{T} \boldsymbol{z}_{1}}_{\leq 0}+k_{3} \boldsymbol{z}_{1}^{T} \boldsymbol{Q} \boldsymbol{z}_{2} .
$$

We now continue with the design of the outer-loop controller, as depicted in Fig. 4. For the derivative of (21), we obtain

$$
\dot{\boldsymbol{z}}_{2}=\left({ }^{b} \dot{\boldsymbol{\omega}}{ }^{i} \dot{\boldsymbol{v}}\right)^{T}-\dot{\boldsymbol{\alpha}}
$$

which we can rewrite using (11) to

$$
\boldsymbol{M}_{u a v} \dot{z}_{2}=\boldsymbol{a}_{u a v}+\overline{\boldsymbol{B}}_{u a v} \boldsymbol{u}-\boldsymbol{M}_{u a v} \dot{\boldsymbol{\alpha}} .
$$

A possible Lyapunov function candidate for the closed-loop system is then

$$
V_{2}=V_{1}+\frac{1}{2} \boldsymbol{z}_{2}^{T} \boldsymbol{M}_{u a v} \boldsymbol{z}_{2}>0
$$

which is positive definit and whose first derivative with respect to time is

$$
\dot{V}_{2}=\dot{V}_{1}+\boldsymbol{z}_{2}^{T} \boldsymbol{M}_{\text {uav }} \dot{\boldsymbol{z}}_{2} .
$$

Inserting (25) in (27) yields

$$
\begin{aligned}
\dot{V}_{2}= & \dot{V}_{1}+\boldsymbol{z}_{2}^{T} \boldsymbol{a}_{u a v}+\boldsymbol{z}_{2}^{T} \overline{\boldsymbol{B}}_{u a v} \boldsymbol{u}-\boldsymbol{z}_{2}^{T} \boldsymbol{M}_{u a v} \dot{\boldsymbol{\alpha}} \\
= & -k_{3} \boldsymbol{z}_{1}^{T} \boldsymbol{Q} \boldsymbol{K}_{1} \boldsymbol{Q}^{T} \boldsymbol{z}_{1}+k_{3} \boldsymbol{z}_{1}^{T} \boldsymbol{Q} \boldsymbol{z}_{2}+\boldsymbol{z}_{2}^{T} \boldsymbol{a}_{u a v} \\
& +\boldsymbol{z}_{2}^{T} \overline{\boldsymbol{B}}_{u a v} \boldsymbol{u}-\boldsymbol{z}_{2}^{T} \boldsymbol{M}_{u a v} \dot{\boldsymbol{\alpha}} .
\end{aligned}
$$

Our aim is to render (28) negative definit, hence, we derive the backstepping control law as

$$
\boldsymbol{u}=\overline{\boldsymbol{B}}_{u a v}^{-1}\left(-\boldsymbol{K}_{2} \boldsymbol{z}_{2}-\boldsymbol{a}_{u a v}+\boldsymbol{M}_{u a v} \dot{\boldsymbol{\alpha}}-k_{3} \boldsymbol{Q}^{T} \boldsymbol{z}_{1}\right)
$$

wherein $\boldsymbol{K}_{2}>0$ is another positive definit diagonal gain matrix. Finally, inserting (29) into (28) yields the Lyapunov function for the complete system (11) under the controller (29)

$$
\dot{V}_{2}=-k_{3} \boldsymbol{z}_{1}^{T} \boldsymbol{Q} \boldsymbol{K}_{1} \boldsymbol{Q}^{T} \boldsymbol{z}_{1}-\boldsymbol{z}_{2}^{T} \boldsymbol{K}_{2} \boldsymbol{z}_{2} \leq 0,
$$

with $\boldsymbol{K}_{1}>0, \boldsymbol{K}_{2}>0$ and $k_{3}>0$. Note, that the quaternion part of $\boldsymbol{Q}$ is an additional state-dependent gain matrix. Asymptotic stability of the equilibrium $\boldsymbol{r}_{e}=\mathbf{0}$, $\boldsymbol{q}_{e}=\left(\begin{array}{llll}1 & 0 & 0 & 0\end{array}\right)^{T},{ }^{b} \boldsymbol{\omega}={ }^{i} \boldsymbol{v}=\mathbf{0}$ can be shown by applying the LaSalle invariance theorem ([12], p. 128).

Remark 1: Due to

$$
\boldsymbol{Q}^{T} \boldsymbol{z}_{1}=\left[\overline{\operatorname{sgn}}\left(\eta_{e}\right) \boldsymbol{\epsilon}_{e} \quad-\boldsymbol{I}_{3 \times 3} \boldsymbol{r}_{e}\right]^{T}
$$

the control $\boldsymbol{u}$ can jump at the transition from $\eta_{e}>0$ to $\eta_{e}<0$ and vice versa. Asymptotic stability can therefore only be guaranteed locally for $\eta_{e} \neq 0$.

Remark 2: For implementing the controller (29), the derivative of the intermediate control law (22) with respect to time can be computed from attitude, position, and velocity measurements as

$$
\begin{aligned}
\dot{\boldsymbol{\alpha}} & =-\boldsymbol{K}_{1}\left(\dot{\boldsymbol{Q}}^{T} \boldsymbol{z}_{1}+\boldsymbol{Q}^{T} \dot{\boldsymbol{z}}_{1}\right) \\
& =-\boldsymbol{K}_{1}\left(\left(\frac{\partial \boldsymbol{Q}^{T}}{\partial \eta} \dot{\eta}+\frac{\partial \boldsymbol{Q}^{T}}{\partial \boldsymbol{\epsilon}} \dot{\boldsymbol{\epsilon}}\right) \boldsymbol{z}_{1}+\boldsymbol{Q}^{T} \boldsymbol{Q}\left(\begin{array}{c}
{ }^{b} \boldsymbol{\omega} \\
{ }^{i} \boldsymbol{v}
\end{array}\right)\right) .
\end{aligned}
$$

\section{Extended Cartesian Impedance Controller}

The backstepping controller (29) presented above generates a torque $\Delta \boldsymbol{\tau}_{a t t}=\boldsymbol{u}_{1}$ that is sent to the VTOL UAV and a force ${ }^{i} \boldsymbol{F}_{E E}=\boldsymbol{u}_{2}$ that should be applied by the manipulator's end-effector to the UAV. The latter follows from (29) as

$$
\begin{aligned}
{ }^{i} \boldsymbol{F}_{E E} & =\left(\boldsymbol{K}_{1} \boldsymbol{K}_{2}+k_{3} \boldsymbol{I}\right) \boldsymbol{r}_{e}-\left(m \boldsymbol{K}_{1}+\boldsymbol{K}_{2}\right){ }^{i} \boldsymbol{v} \\
& +\underbrace{m g \boldsymbol{e}_{3}-f_{T} \boldsymbol{R}_{i b} \boldsymbol{e}_{3}}_{\boldsymbol{F}_{F T S, F z}} .
\end{aligned}
$$

Note that since the thrust force $f_{T}$ of a VTOL UAV is not directly measured in practice, it is convenient to use the force 
$\boldsymbol{F}_{F T S, F_{z}}$ measured by the force-torque sensor (FTS) at the end-effector for feed-back control.

The force (33) can be mapped to robot joint torques, as presented in Section II for the classical Cartesian impedance controller (6). Similar to (5), we can define a dynamical relationship for the forces at the end-effector as

$$
\boldsymbol{\Lambda}(\phi) \ddot{\boldsymbol{x}}_{e}+\boldsymbol{\mu}(\boldsymbol{\phi}, \dot{\boldsymbol{\phi}}) \dot{\boldsymbol{x}}_{e}-\left({ }^{i} \boldsymbol{F}_{E E} \quad \boldsymbol{\tau}_{\text {ori }}\right)^{T}=\boldsymbol{F}_{\text {ext }}
$$

and derive the torque control law for the robot manipulator

$$
\begin{aligned}
& \boldsymbol{\tau}_{j}=\boldsymbol{g}(\boldsymbol{\phi})+\boldsymbol{\tau}_{n s p}+\mathcal{J}(\phi)^{T}\left(\boldsymbol{\Lambda}(\phi) \ddot{\boldsymbol{x}}_{d}+\boldsymbol{\mu}(\boldsymbol{\phi}, \dot{\boldsymbol{\phi}}) \dot{\boldsymbol{x}}_{d}\right) \\
& +\mathcal{J}(\phi)^{T}\left({ }^{i} \boldsymbol{F}_{E E} \quad \boldsymbol{\tau}_{\text {ori }}\right)^{T} .
\end{aligned}
$$

The different sign of the last term in (35) compared to (6) results from the definition of the position error $\boldsymbol{r}_{e}$. Using the input $\tau_{\text {ori }}$, the orientation of the end-effector can be controlled independently from the orientation of the UAV. Furthermore, the term $\boldsymbol{\mu}(\phi, \dot{\boldsymbol{\phi}}) \dot{\boldsymbol{x}}_{d}$ in (35) is neglected in the practical implementation due to its minor numerical values. The positive diagonal matrices $\boldsymbol{K}_{1}$ and $\boldsymbol{K}_{2}$ as well as the scalar $k_{3}$ are the design parameters of the extended Cartesian impedance controller.

\section{EXPERIMENTAL RESULTS}

In order to validate our proposed control strategy, we perform experiments using the demonstrator introduced in Section I and the off-the-shelf quadrotor AR.Drone 2.0 [18]. The model parameters for the quadrotor can be found in Table I. The quadrotor is connected to the end-effector of the robot via the universal hinge shown in Fig. 1. All controllers are implemented using MATLAB/Simulink and the communication with the AR.Drone is established via standard $2.4 \mathrm{GHz}$ wireless LAN.

We evaluate both the performance of the orientation control of the VTOL UAV and the position control of the robot manipulator. The results of three experiments, which are summarized in Table II, are presented in the following. For all experiments, the same desired position trajectory with respect to time $t$, shown in Fig. 5, is used and the desired orientation of the quadrotor is set to $\boldsymbol{q}_{d}=\left(\begin{array}{llll}1 & 0 & 0 & 0\end{array}\right)^{T}$. The gains of the backstepping controller found by trial-and-error are $\boldsymbol{K}_{1}=\boldsymbol{K}_{2}=\left[\begin{array}{lll}\boldsymbol{I}_{3 \times 3} & 30 \cdot \boldsymbol{I}_{3 \times 3}\end{array}\right]^{T}$ and $k_{3}=35$. For the implementation of the controller (35) we use a gravity compensation $\boldsymbol{g}(\phi)$ and an elbow-field to compute $\boldsymbol{\tau}_{n s p}$ [19] such that the elbow of the robot points downwards. We want the end-effector to always point in positive ${ }^{i} z$-direction and use a proportional-derivative (PD) controller with gains $k_{p}=25$ and $k_{d}=7$ to generate the torque $\boldsymbol{\tau}_{\text {ori }}$ for the end-effector orientation.

The initial experiment E1 sets the basis for comparison. Therefore, we neither use the additional torque $\Delta \boldsymbol{\tau}_{\text {att }}$ nor the measurement from the force-torque sensor $\boldsymbol{F}_{F T S}$. The desired acceleration of the end-effector $\ddot{\boldsymbol{x}}_{d}$ is also set to zero. The results of E1 reveal overshoots in the end-effector position (see Fig. 6a) and a deviation of up to 9.2 deg from the desired orientation, illustrated in Fig. 7a using Euler angles $\left(\begin{array}{lll}\varphi & \theta & \psi\end{array}\right)^{T}=\left(\begin{array}{lll}0 & 0 & 0\end{array}\right)^{T}$.
TABLE I: Model parameters of the quadrotor AR.Drone 2.0

\begin{tabular}{c|c|c} 
Mass $m[\mathrm{~kg}]$ & Inertia $\boldsymbol{J}\left[\mathrm{kg} \mathrm{m}^{2}\right]$ & Hinge length $l[\mathrm{~m}]$ \\
\hline \hline 0.480 & $\operatorname{diag}([0.0060 .0070 .012])$ & 0.06 \\
\hline
\end{tabular}

TABLE II: Summary of the presented experiments

\begin{tabular}{c|l} 
Experiment & Conditions \\
\hline \hline E1 & $\Delta \boldsymbol{\tau}_{a t t}=\mathbf{0}, \ddot{\boldsymbol{x}}_{d}=\mathbf{0}, \boldsymbol{F}_{F T S}=\mathbf{0}$ \\
E2 & Using $\Delta \boldsymbol{\tau}_{a t t}$ and $\ddot{\boldsymbol{x}}_{d} ; \boldsymbol{F}_{F T S}=\mathbf{0}$ \\
E3 & Using $\Delta \boldsymbol{\tau}_{a t t}, \ddot{\boldsymbol{x}}_{d}$, and $\boldsymbol{F}_{F T S}$ \\
\hline
\end{tabular}

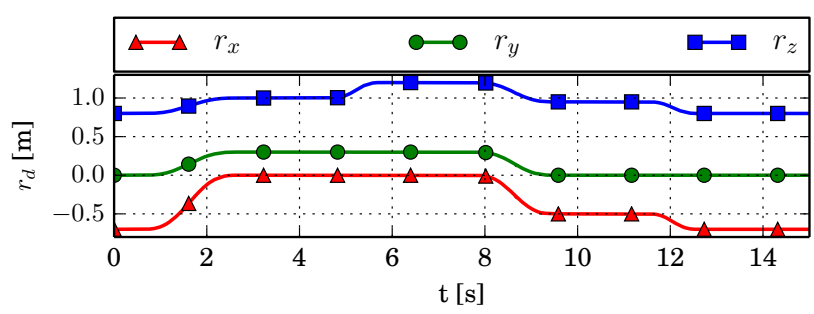

Fig. 5: Desired trajectory $\boldsymbol{r}_{d}(t)$ for experiments E1, E2, and E3.

In the second experiment E2, the desired acceleration $\ddot{\boldsymbol{x}}_{d}$ is used in the controller (35) and the torque $\Delta \boldsymbol{\tau}_{a t t}$, depicted in Fig. 7c, is sent to the UAV. Fig. 6a shows that using the acceleration $\ddot{x}_{d}$ leads to an increased accuracy in position tracking with less overshoot, while there is still a deviation from the desired trajectory. Fig. $7 b$ shows a decreased deviation from the desired orientation compared to E1, but the amplitude is still up to $6.8 \mathrm{deg}$. This is due to the fact that the torque of the AR.Drone is feed-forward controlled which does not yield accurate results.

In the final experiment E3, the force $\boldsymbol{F}_{F T S, F_{z}}$ measured by the force-torque sensor (Fig. 6b) is additionally fed back into (33). The results of E3, depicted in Fig. 6a, indicate that the forces acting at the robotic end-effector in ${ }^{i} z$-direction are better compensated and that therefore the controller's performance in position tracking in this direction is increased. The torque $\Delta \boldsymbol{\tau}_{a t t}$ in E3 was similar to E2 (see Fig. 7c) and is therefore omitted here.

Hence, it can be concluded that the backstepping controller (29) combined with the extended impedance controller (35) enables compliant and coordinated physical interaction between the robot manipulator and the VTOL UAV with decent accuracy for the landing task.

\section{CONCLUSION AND FUTURE WORK}

In this paper, a novel landing system for VTOL UAVs is presented. The system uses a robot manipulator to assist a UAV in the last landing phase by physical interaction. With this support, a UAV rotorcraft should be able to land safely under severe conditions, where the landing of the same UAV without any support is hardly possible. We present an analytical analysis of such type of system by modeling UAV and manipulator as two subsystems and design a nonlinear backstepping controller. Our approach extends the classical Cartesian impedance controller to account for the UAV's rigid-body dynamics and allows for coordinated control of VTOL UAV and manipulator. Experimental results demonstrate the applicability of the proposed control strategy. 

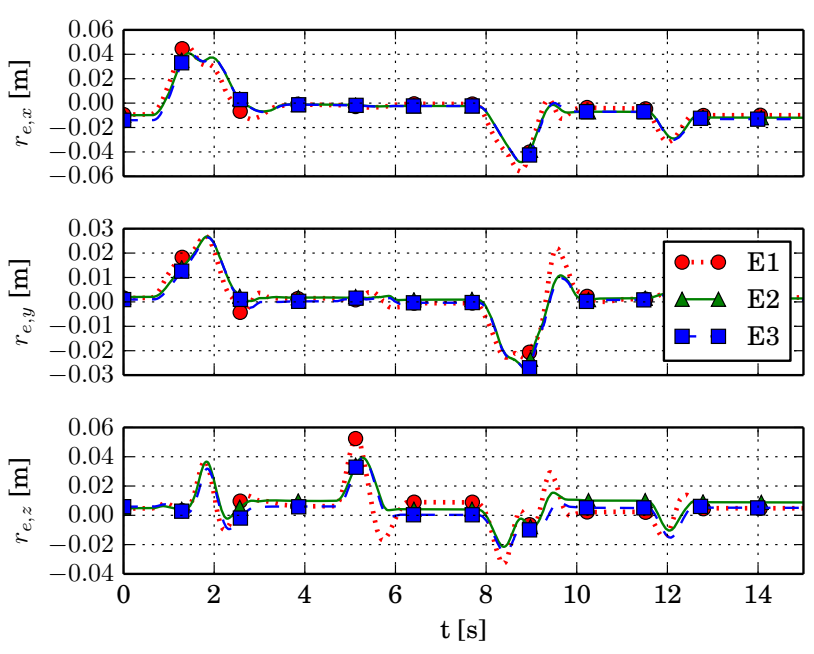

(a) Position errors $\boldsymbol{r}_{e}(t)$ in experiments E1, E2, and E3.

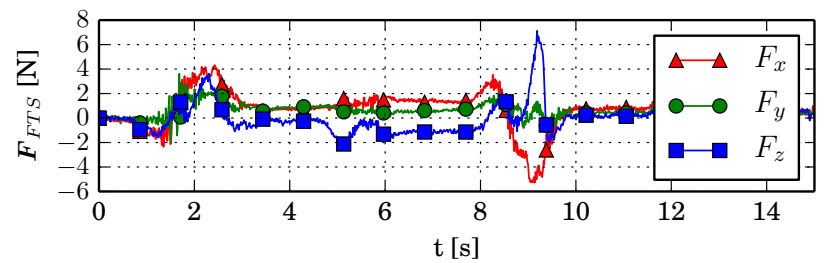

(b) FTS measurement used in E3 to increase the tracking performance.

Fig. 6: Position errors in experiments E1-E3 (top) and force sensor measurement in experiment E3 (bottom).

In the next step, we are going to incorporate actuator limits of the aerial vehicle, disturbance observation, and compensation of platform movements, e.g. ship motions, in the control approach. Moreover, we plan to develop strategies for approaching the manipulator and for autonomously establishing the actual connection between the VTOL UAV and the robot manipulator's end-effector.

\section{REFERENCES}

[1] T. Tomic, M. Maier, and S. Haddadin, "Learning quadrotor maneuvers from optimal control and generalizing in real-time," in Proc. IEEE Int. Conf. on Robotics and Automation (ICRA), May 2014.

[2] J. Forrest, I. Owen, G. Padfield, and S. Hodge, "Ship-helicopter operating limits prediction using piloted flight simulation and timeaccurate airwakes," J. of Aircraft, vol. 49, no. 4, pp. 1020-1031, July 2012. [Online]. Available: http://dx.doi.org/10.2514/1.C031525

[3] J. Hervas, M. Reyhanoglu, and H. Tang, "Automatic landing control of unmanned aerial vehicles on moving platforms," in Proc. IEEE Int. Symp. on Industrial Electronics (ISIE), June 2014, pp. 69-74.

[4] A. Isidori, L. Marconi, and A. Serrani, "Robust nonlinear motion control of a helicopter," IEEE Trans. on Automatic Control, vol. 48, no. 3, pp. 413-426, March 2003. [Online]. Available: http://dx.doi.org/10.1109/TAC.2003.809147

[5] L. Sandino, M. Bejar, and A. Ollero, "On the applicability of linear control techniques for autonomous landing of helicopters on the deck of a ship," in Proc. IEEE Int. Conf. on Mechatronics (ICM), April 2011, pp. 363-368.

[6] B. Herisse, T. Hamel, R. Mahony, and F.-X. Russotto, "The landing problem of a vtol unmanned aerial vehicle on a moving platform using optical flow," in Proc. IEEE/RSJ Int. Conf. on Intelligent Robots and Systems (IROS), Oct 2010, pp. 1600-1605.

[7] J. Sanchez-Lopez, S. Saripalli, P. Campoy, J. Pestana, and C. Fu, "Toward visual autonomous ship board landing of a VTOL UAV," in Proc. Int. Conf. on Unmanned Aircraft Systems (ICUAS), May 2013, pp. $779-788$.

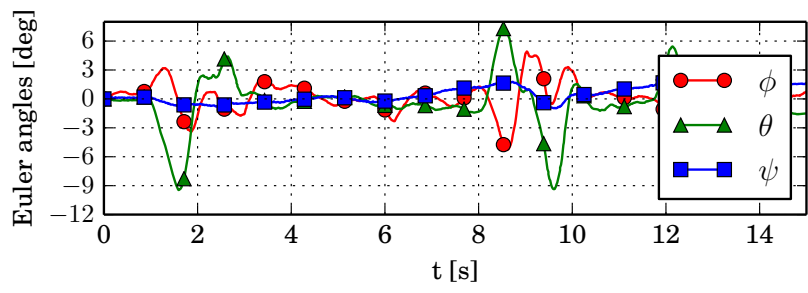

(a) Orientation of the AR.Drone quadrotor in experiment E1.

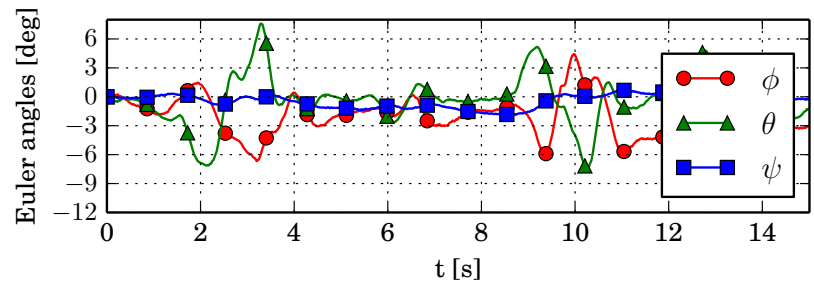

(b) Orientation of the AR.Drone quadrotor in experiment E2.

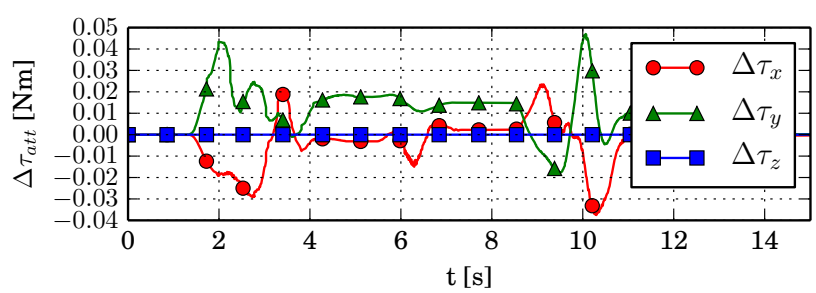

(c) Torque $\Delta \boldsymbol{\tau}_{\text {att }}$ sent to the AR.Drone quadrotor in experiment E2.

Fig. 7: Orientation of the quadrotor in E1 (top) and in E2 (middle), as well as the torque $\Delta \boldsymbol{\tau}_{\text {att }}$ sent to the AR.Drone in E2 (bottom).

[8] A. Campos, J. Quintero, R. Saltaren, M. Ferre, and R. Aracil, "An active helideck testbed for floating structures based on a stewart-gough platform," in Proc. IEEE/RSJ Int. Conf. on Intelligent Robots and Systems (IROS), Sept 2008, pp. 3705-3710.

[9] S.-R. Oh, K. Pathak, S. Agrawal, H. Pota, and M. Garratt, "Approaches for a tether-guided landing of an autonomous helicopter," IEEE Trans. on Robotics, vol. 22, no. 3, pp. 536-544, June 2006.

[10] L. Sandino, D. Santamaria, M. Bejar, A. Viguria, K. Kondak, and A. Ollero, "Tether-guided landing of unmanned helicopters without GPS sensors," in Proc. IEEE Int. Conf. on Robotics and Automation (ICRA), May 2014, pp. 3096-3101.

[11] C. Ott, Cartesian Impedance Control of Redundant and Flexible-Joint Robots. Springer Science \& Business Media, Aug 2008.

[12] H. K. Khalil, Nonlinear systems. Prentice Hall, Inc, 2002.

[13] O. Khatib, "Inertial properties in robotic manipulation: An object-level framework," The Int. Journal of Robotics Research, vol. 14, no. 1, pp. 19-36, 1995.

[14] K. Kondak, M. Bernard, N. Meyer, and G. Hommel, "Autonomously flying vtol-robots: Modeling and control," in Proc. IEEE Int. Conf. on Robotics and Automation (ICRA), April 2007, pp. 736-741.

[15] P. De Monte and B. Lohmann, "Trajectory tracking control for a quadrotor helicopter based on backstepping using a decoupling quaternion parametrization," in Proc. Mediterranean Conf. on Control and Automation (MED), June 2013, pp. 507-512.

[16] R. Kristiansen, P. Nicklasson, and J. Gravdahl, "Satellite attitude control by quaternion-based backstepping," IEEE Trans. on Control Systems Technology, vol. 17, no. 1, pp. 227-232, Jan 2009.

[17] S. Bhat and D. Bernstein, "A topological obstruction to global asymptotic stabilization of rotational motion and the unwinding phenomenon," in Proc. American Control Conference, vol. 5, June 1998, pp. 2785-2789.

[18] P.-J. Bristeau, F. Callou, D. Vissiere, N. Petit et al., "The navigation and control technology inside the ar.drone micro uav," in 18th IFAC world congress, vol. 18, no. 1, 2011, pp. 1477-1484.

[19] F. Huber, K. Kondak, K. Krieger, D. Sommer, M. Schwarzbach, M. Laiacker, I. Kossyk, S. Parusel, S. Haddadin, and A. Albu-Schaffer, "First analysis and experiments in aerial manipulation using fully actuated redundant robot arm," in Proc. IEEE/RSJ Int. Conf. on Intelligent Robots and Systems (IROS), Nov 2013, pp. 3452-3457. 